\title{
Health Care for an Aging Society on the Belt and Road: A Zhejiang Story
}

\author{
Shiming Tang, Changjuan Guo*, Wenjie Ge, Lidan Xu \\ Hangzhou Normal University \\ Hangzhou, Zhejiang, China \\ *Email: 836543186@qq.com
}

\begin{abstract}
Purpose: Meeting challenges and demands of society of aging, population of advanced aging and elderly families and innovating the home care service. Methods: Research group selected 25 communities under the jurisdiction of Tianshui Street, Changqing Street and Xiaoying Street in Hangzhou as the typical communities and used literature research method, historical research method, comparative research method, investigation method and so on to carry out research. Results: The typical community home care service possesses the scientificity of the earlier work, the feasibility of better solving problems and the creativity of creating the brand successfully. Conclusion: Research Group analysed the experience of Hangzhou typical communities, puts forward relevant policy suggestions to solve the three problems which are "poor and sickness occurring in aging families simultaneously" and "community resource shortage" and "the quality imbalance of professional cares", realizing the work innovation of "family centered", "community oriented", "vocational services founded".
\end{abstract}

Keywords: Society of Aging, the Belt and Road, Zhejiang Stories, Health Care Hub

\section{INTRODUCTION}

Most of the western developed countries are "getting rich before they are old". They have entered the aging stage under the background of basically realizing modernization. However, Chinese society is facing multiple challenges of society of aging, population of advanced aging and family with solitary empty-nest status in the relatively backward grasslands that have not yet achieved modernization. Despite more than 10 years' study, Dalian, Shanghai, Ningbo and other places have explored a "nursing home in family", "purchasing home-based care services by government", "non-profit organizations' aiming for the home-based care service" and other ways to solve the problem of "endowment difficulty", but can not meet the real needs of elderly. There are three major problems in the community service for the elderly. Firstly, "family tied" is difficult; Secondly, "community oriented" is difficult; Thirdly, "vocational services founded" is difficult.

\section{II.INTRODUCE A QUESTION}

From the traditional family pension to the welfare institution endowment, then returning to the community, family and home for the aged is the experience destination and development track for most developed countries to solve the problem of "endowment difficulty". In recent years, many developed countries have shown the obvious trend of the decrease in the number of institutions for the aged and the increase in the number of houses for the aged. The burden of Institutional pension is huge, and it can not solve the problem of "endowment difficulty". It is necessary to improve the service of the community for the aged as soon as possible.

The main project of the home care service in developed countries is to take care of the basic daily life of elderly, and the volunteers have taken the services of entertainment and education. The UK is taking a model of community care, and Denmark has launched an independent pension community. Canada has implemented the government's purchase of the home pension plan for the whole people. Germany emphasizes the mutual assistance of old and college students, while Japanese government emphasizes that "let elderly separate from hospital, return to the community and family". American home-based care service is industrialized and the elderly are managed by experts' team of health center and multidisciplinary.

The home care service in China is a dollop of socialized provision, which is mainly provided by the elderly at home to solve daily life problems. There are two main forms of service: door-to-door service provided by professional training attendants and the elderly day service created by community. It is worth mentioning that in the context of the accelerated aging of aging, the accelerated expansion of urbanization, and the acceleration and advancement of empty nesters, home care services in typical communities in Hangzhou have advantages of both the scientific nature of earlier work, the feasibility of better solving problems and the creativity of creating the brand successfully.

\section{CASE OF TYPICAL COMMUNITY IN HANGZHOU}

Research Group selected 25 communities under the jurisdiction of Tianshui Street, Changqing Street and Xiaoying Streets in Hangzhou as the typical communities. The typical communities possess the area of 5.46 square kilometers, a population of 187 thousand and 200 Cangqiao Community, Wangma Community and Xiaoying Community become demonstration communities of work innovation. Cangqiao Community is a demonstration service window for empty nest service in large and medium-sized cities of China. It is also a standardized community in Zhejiang Province, and an advanced community service in China. Cangqiao Community is a demonstration service window for empty nest service in large and medium-sized cities of China. It is also a 
standardized community in Zhejiang Province, and an advanced community service in China. In 2007, General Secretary $\mathrm{Hu}$ Jintao inspected Wangma Community and highly appraised the first corridor Party branch in China established by this community.

The sparrow may be small but fully-equipped. The following two prominent phenomena have been discovered through literature research, historical research, comparative research, investigation and so on.

\section{A. Institutions Are in Short Supply for the Aged}

At present, the population of elderly in typical communities is close to $20 \%$, and the old people in the province account for about 15\%. In 2030 and 2040, Hangzhou and Zhejiang are expected to break through $35 \%$ and 1/3 respectively. At the same time, the proportion of empty nest living alone in urban elderly households is rising. Compared to $1 / 3$ of some cities in China, the proportion in Hangzhou lakeside and other streets is more than doubled. It is expected that by 2015, the proportion of empty nesters living alone in urban households can reach $90 \%$. According to statistics, the national old-age welfare institutions are in short supply and can only accept about $2 \%$ of the total.

Taking Tianshui Street as an example, nearly 40000 of the residents, nearly 9000 of the elderly, nearly 900 elderly needs help. Other typical communities are also roughly the same, about $20 \%$ of the elderly with diseases and difficulties need to be taken care of. It is difficult to solve the problem of elderly by relying on institutions.

\section{B. Home Care Needs Help}

Research found that elderly people in typical communities generally suffering from senile disease, of which $70 \%$ have chronic diseases and $15 \%$ suffer from serious illness. Most people are irreversible in the need for life-long treatment and care. The daily living of the old people need daily help and care, otherwise accidents will occur. The old man is also generally suffering from mental illness, $40 \%$ of them suffering from depression. With living in narrow circle, the aged often worry about personal gains and losses, and feel disappointed. In addition to the physical and mental disorders, widows, orphans and elderly women generally have low income which seriously influences health protection, medical care and other necessary expenses.

In summary, the current pension problem can be interpreted as three major problems: firstly, "family tied" is difficult and the focus is "poor and sickness occurring in aging families simultaneously". The proportion of elderly people living alone in the structure of family members has increased greatly which makes the problem more serious. Secondly, "community oriented" is difficult and the focus is "the resource community is in short supply", which is generally shown in the limited community resources and difficult to develop. Thirdly, "vocational services founded" is difficult and the focus is "the quality imbalance of professional cares". It is generally manifested in the lack of professional quality of maintenance personnel, and the reality also leads to "labor shortage".

\section{CONCLUSIONS AND SUGGESTIONS}

\section{A. Through Analysis of the Experience of Typical Communities in Hangzhou, We can Draw the Following Conclusions:}

1) Solving the Problem of "Poor and Sickness Occurring in Aging Families Simultaneously" through Special Government Support, Neighborhood Arrangement and Helping Home Care for the Aged

In order to promote home care, various projects such as "community networking service", "residents self help" and "service time access" have been promoted everywhere, which is beneficial but limited. Typical communities in Hangzhou have assured the elderly daily life support intervention by striving for special government support, have scientifically planned psychological intervention in physical and mental disorders and emergency assistance through coordination of all levels of organizations, have realized the best integration of resources of human, money and material through the innovation of community work.

Under the premise of respecting the desire of the elderly at home, we should pursuit to develop scientifically. The operation should be economical and efficient, and the service guarantee should be sustained and feasible. Wangma community created socialized services called "66810 service for people". The service under the leadership of community Party committee, coordinating with community neighborhood committees, residents and jurisdiction units, meeting the multi-level need of residents, relying on streets and community residents committee. It includes four parts: "six must come", "six must visit", "eight must report" and "ten lines for public service" which speciality includes home care line.

2) Solving the Problem of "Community Resource Shortage" by Organizing Talent Team, Improving the Governing System and Striving for Support

Organizing the talent team should be good at using local materials. Typical communities in Hangzhou firstly give full play to the backbone role of social workers in the street, community and corridor, and fully mobilize the residents of talent to strengthen the quality of home care service. The relevant streets and community organizations also work together to do a good job of scheduling, communicating, visiting, consulting, and so on. Typical communities accomplish the network support based on organizations, information storage based on archives, deep promotion based on activities, high effectiveness based on system. The fund-raising of related funds is more proactive with having a budget at the beginning of the year and a balance at the end of the year. As the open system and the effective operation, the street finance and the neighbourhood of the community are strongly supportive. Taking Cangqiao community as an example, the street provides more than 200 million yuan at a time to support the new pension club, ensuring the activities and friendship of the elderly.

3) Solving the Problem of "the Quality Imbalance of Professional Cares" by Strengthening Grass-roots Construction, Integrating Voluntary Service and Providing Round-the-clock Service 
The construction of organizations should go deep roots. For example, seven demonstration communities of Tianshui Street have established grassroots organizations, with community instructors, building workers and corridor liaisons. The organizations build a "worry-free network" that provide 24-hour routine help and emergency rescue. That is daily meeting, daily record, daily door-to-door, daily service, etc. It is also a seamless connection to social public welfare activities. Businesses and institutions in the area where the community is located also provide targeted services.

\section{B. Accordingly, We Propose the following Policy Recommendations:}

1) Innovating the Carrier of Home Care Service and Realizing "Family Centered"

In recent years, with the support of local governments, the typical communities has improved the service of home care for the aged by implementing the work innovation of "eight one" and other service carriers. The "eight one" includes concretely: a queried file table for implement dynamic management; a notified monitoring network for solving practical problems; a responsive help line for providing hotline service; a reliable mediating agent for coordinating timely; a responsible service team for perfecting volunteer service; a sensitive beeper for providing home service; a warm chat room for comforting and communicating; a multi-functional activity room for enriching cultural life.

Taking the "eight one" service as an example, Hangzhou community work integrates the latest achievements of social demography, public administration, aging behavior, ergonomics and many other disciplines, exploring a new home care path which community provides main service guarantee. The path is operationally feasible, economically efficient and in line with the physical and mental characteristics and wishes of the elderly.

2) Popularizing the Technological Achievements in Home Care and Realizing" Community Oriented"

Action research based on community fills research blank such as support intervention, psychological intervention and emergency assistance which are needed in home care urgently. It yields twice the result with half the effort. In the face of shortages such as official social workers, scientific management, Supporting sites and funds in place, Hangzhou typical communities establish the "physical and mental health" mechanism of property and system, innovating "emergency assistance green channel" such as "golden key for emergency rescue", "zero threshold for first aid", "application system for special difficulties allowance" and improving the daily assistance system such as getting sick, traveling and get into trouble which are included in "six must visit".

There is a case in typical community. The enterprise retiree can participate in social assistance guarantee by paying for one yuan ever month symbolically. A couple surrendered in the spring and received eight yuan. In autumn, the husband got ill, and the rescue cost 300,000 yuan. The original social help mechanism can be reimbursed $80 \%$, and the difficult assistance can also reimburse $70 \%$ of the balance of 60,000 yuan, which can't be enjoyed One yuan per month can save 282,000 yuan, and surrender can't be enjoyed. When the retired person was in a desperate situation for surrender, the typical community immediately launched the "zero threshold for first aid" to help him handling supplementary insurance, relief and community special assistance. The annual living allowance is more than 2000 yuan. The masses said that community cadres are really more relative than their relatives.

Take the "golden key project" and "zero threshold for first aid" as an example of community emergency assistance in Cangqiao community. A woman from solitary lesions and the empty nest on the verge of death or destruction, helping pair after the discovery, immediately use the "golden key" in the emergency rescue. One empty nest alone woman is in danger because of pathological changes, help the child to discover the situation, immediately enable "golden key" to break door into emergency rescue.

A woman who lives alone is in danger for disease. Her helping pair discovered it and immediately used the "golden key" to break into for emergency rescue. After the old man recovered, she said "it was the community pension system that gave me second life".

Therefore, only introducing innovative technology, grasping the law of experience and promoting scientific management can truly implement "community oriented".

3) Promoting Sustainable Development of the Region and Realizing" Vocational Services Founded"

Through innovating work, the typical communities in Hangzhou realize the problem solving, realizing "service resources", "professional guidance", "scientific division of labor", "special intervention" and "entering into posts" relying on professional services, and have opened up a new path of home care service.

Finally, the typical communities built a brand based on professional service by identifying "combination of production and learning" and "international cooperation", optimizing and adjusting the service system for the aged, transforming and upgrading the pension service industry, promoting the high-tech research and development of special products for the elderly, and leading to regional tourism and leisure, ecological residence, cultural creativity, technological innovation, information consultation, financial business, and even the economic growth in aging era.

\section{ACKNOWLEDGEMENT}

This paper was a particial achievement and financially supported by the All-China Federation of Returned Overseas Chinese Project (17DZQK203)“Zhejiang Stories on the Belt and Road People's Connectivity" and the 4th Academic Conference Project for Zhejiang Provincial Federation of Social Sciences (Sub-forum 201830) "Zhejiang Hub Construction for the Belt and Road".

\section{REFERENCES}

[1] Tang Shiming. The creation and demonstration of Urban Empty Nest Service Guarantee Mechanism, J. Chinese Journal of 
Gerontology. 20 (2008) 2046-2047.

[2] Tang Shiming. Establishment and demonstration of service guarantee mechanism for Hangzhou empty nest elderly people -an action study on the aging of strain society in Hangzhou, J.

Journal of Zhejiang Gongshang University. 04 (2008)65-68. 